\title{
Dendritic cell stimulation by mycobacterial Hsp70 is mediated through CCR5
}

\author{
Maurizio Vignola Award on Innovation in Pneumology, supported by an unrestricted grant from the \\ Chiesi Foundation
}

\section{Rodrigo Andres Floto}

Respiratory Infection, Inflammation and Immunology Section, Papworth Hospital, Cambridge, and Cambridge Institute for Medical Research, University of Cambridge, Cambridge, UK.

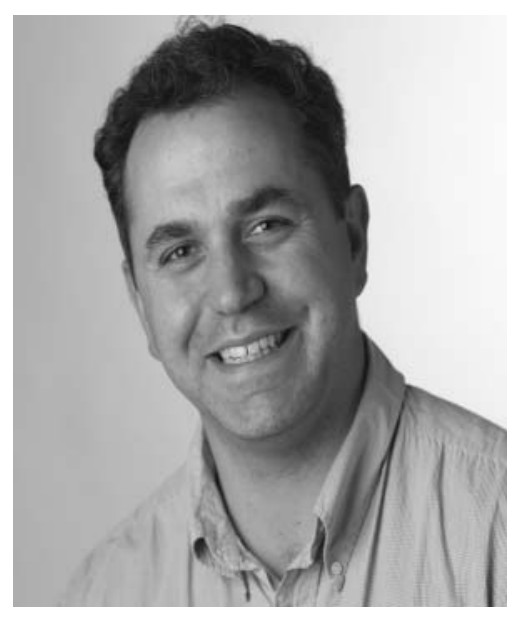

It is a great honour to have received this award, which is dedicated to the memory of the outstanding academic physician, Maurizio Vignola.

I graduated from the MB-PhD programme at the University of Cambridge (Cambridge, UK) in 1997, undertook clinical training at the Royal Brompton and Hammersmith Hospitals (both London, UK) and Papworth Hospital (Cambridge). Since 2002, I have worked at the Cambridge Institute for Medical Research (Cambridge), supported by the UK Medical Research Council. I combine laboratory research with clinical work as part of the Respiratory Infection, Inflammation and Immunology service at Papworth Hospital caring for patients with cystic fibrosis (CF), non-CF bronchiectasis and immune-mediated lung disease.

My research interests have focused on understanding the basic mechanisms of receptor control of macrophage and dendritic cell (DC) function in order to elucidate the pathophysiology of both microbial (particularly mycobacterial) lung infection and autoimmune lung disease [1,2]. As the dedicated antigenpresenting cells of the immune system, DCs and macrophages must link the innate to the adaptive immune response. One of their critical, but poorly understood, functions is the

STATEMENT OF INTEREST: None declared. appropriate handling of ingested antigens; specifically whether to reinforce immune tolerance or initiate immune activation. Internalisation can result in safe, nonimmunogenic clearance that represents an appropriate response to self-antigen. Failure of this process may initiate autoimmunity. Alternatively, internalisation may lead to immunogenic antigen presentation to $\mathrm{T}$ and B-cells; the appropriate host response to pathogens. This process may, however, fail in some bacterial infections and predispose to bronchiectasis.

By defining how antigen processing by DCs and macrophages is normally controlled and sometimes deregulated, I have been able to start assessing the effect of its therapeutic manipulation in pulmonary autoimmunity and mycobacterial infection.

The paper I submitted for the Maurizio Vignola Award [1] was the result from a long-standing collaboration with Paul Lehner (Cambridge Institute for Medical Research, University of Cambridge), which examined how DCs are stimulated by a mycobacterial heat shock protein, myHSP70, through its action on the HIV co-receptor CCR5.

Mycobacterium tuberculosis infects one-third of the world's population and is responsible for two million deaths per year. An effective host immune response to mycobacterial infection must control pathogen dissemination without inducing immunopathology, although how this is achieved is poorly understood. One mycobacterial factor that has been implicated is myHSP70. We have previously shown that myHSP70 both efficiently delivers peptide antigen to human DCs and stimulates them to generate effective $\mathrm{T}$-cell functional responses [3]. However, neither the surface receptor nor the cellular mechanisms responsible for these effects have, until now, been identified.

Through pharmacological dissection of myHSP70 signalling and a heterologous functional screening of candidate receptors, we have established that myHSP70 signals through the chemokine receptor, CCR5 [1]. We have confirmed these findings in human DCs by demonstrating that the CCR5specific inhibitor TAK-779 blocks myHSP70 activity while DCs from subjects homozygous for the CCR5 $\Delta 32$ inactivating mutation fail to respond to myHSP70. CCR5-dependent myHSP70 signalling leads to rapid calcium-dependent morphological 
changes and integrin activation, resulting in DC aggregation, the formation of DC-T-cell immune synapses and the generation of effector immune responses. We have found that CCR5 signalling has an important role in controlling mycobacterial infection in vitro.

We conclude that CCR5 acts as a pattern-recognition receptor for myHSP70. These findings have important implications for both our understanding of mycobacterial infection and the use of myHSP70 in tumour-directed dendritic cell immunotherapy.

\section{REFERENCES}

1 Floto RA, MacAry PA, Boname JM, et al. Dendritic cell stimulation by mycobacterial HSP70 is mediated through the HIV co-receptor CCR5. Science 2006; 314: 454-458.

2 Floto RA, Clatworthy MR, Heilbronn KR, et al. Loss of function of a lupus-associated Fc $\gamma$ RIIb polymorphism through exclusion from lipid rafts. Nature Med 2005; 11: 1056-1058.

3 MacAry PA, Javid B, Floto RA, Smith KGC, Singh M, Lehner PJ. HSP70 peptide-binding mutants separate antigen delivery from dendritic cell stimulation. Immunity 2004; 20: 95-106. 